\title{
MRI in Soils: Determination of Water Content Changes Due to Root Water Uptake by Means of a Multi-Slice-Multi-Echo Sequence (MSME)
}

\author{
A. Pohlmeier, ${ }^{*}$, F. Vergeldt ${ }^{2}$, E. Gerkema ${ }^{2}$, H. Van As $^{2}$, D. Van Dusschoten ${ }^{3}$ and H. Vereecken ${ }^{1}$ \\ ${ }^{I} I C G-4$, Research Centre Jülich, Germany \\ ${ }^{2}$ Department Biophysics, Wageningen University, The Netherlands \\ ${ }^{3}$ ICG-3, Research Centre Jülich, Germany
}

\begin{abstract}
Root water uptake by ricinus communis (castor bean) in fine sand was investigated using MRI with multiecho sampling. Before starting the experiments the plants germinated and grew for 3 weeks in a cylindrical container with a diameter of $9 \mathrm{~cm}$. Immediately before the MRI experiments started, the containers were water-saturated and sealed, so water content changes were only caused by root water uptake. In continuation of a preceding work, where we applied SPRITE we tested a multi-echo multi-slice sequence (MSME). In this approach, the water content was imaged by setting $T_{\mathrm{E}}=6.76$ $\mathrm{ms}$ and $n_{E}=128$ with an isotropic resolution of $3.1 \mathrm{~mm}$. We calculated the water content maps by biexponential fitting of the multi-slice echo train data and normalisation on reference cuvettes filled with glass beads and $1 \mathrm{mM} \mathrm{NiCl}{ }_{2}$ solution. The water content determination was validated by comparing to mean gravimetric water content measurements. By coregistration with the root architecture, visualised by a 3D fast spin echo sequence (RARE), we conclude that the largest water content changes occurred in the neighbourhood of the roots and in the upper layers of the soil.
\end{abstract}

Keywords: MRI, root water uptake, root system, relaxometric imaging.

\section{INTRODUCTION}

The interplay between root activity and soil functions is not yet well understood on a local scale, although it belongs to the most important processes controlling plant growth and productivity. One reason is the limited observability under natural conditions, since roots are the "hidden half" of the entire plant [1]. Recently, progress has been made by the development of numerical modelling based on soil physical principles, where water motion is calculated in a coupled soil-plant network of water potential differences, hydraulic conductivities and root system architecture [2-4]. The success of such integrated modelling approaches requires experimental information about the root system architecture as well as water content distributions under controlled boundary conditions in experimental scenarios. Here, non-invasive 3D tomographic methods are mandatory, since only these can give an undisturbed look into the soil and the processes taking place there. Today, three main techniques based on different physical principles are generally usable, X-ray [5], neutron [6] and nuclear magnetic resonance tomography (MRI) [7-10]. The potentials and limits of these methods for investigating root-soil interactions are a present challenging topic in soil and plant sciences.

The focus of X-ray CT is on the physical density with high resolution (some microns), and so this method is highly sensitive for imaging of the solid phase. On the other hand the differentiation between water and air turns out to be

*Address correspondence to this author at the Research Centre Jülich, ICG4 (agrosphere institute), D-52425 Jülich, Germany; Tel: (49)2461-612795; Fax: (49)2461-6125; E-mail: a.pohlmeier@fz-juelich.de tricky. In contrast, MRI is principally capable to image both root system architecture and water content, since its sensitivity is based on the presence of water and on the properties of the medium, in which it is located. Also MRI is capable to image fluxes or water content changes on different time scales.

The present work is a continuation of our previous studies on the use of MRI for the investigation of root water uptake $[10,11]$. There we used a single point imaging technique, since this is especially convenient for matrices with short transversal relaxation times as soils at low water content are [12]. However, single point imaging methods are time consuming since the resolution scales with the cube of the repetition time.

In this work we want to elucidate further the potential of relaxometric imaging for the water content determination [13]. The major concern for such methods is that with decreasing water content the relaxation times decrease strongly according to the Brownstein-Tarr model [14, 15]. So if one just uses conventional spin-echo imaging the echo intensity reflects not only the water content but also the local relaxivity, and it is not necessarily proportional to the water content. As model system we have chosen ricinus communis carmencita (castor bean) grown in medium sand. The scenario was initial saturation and subsequent increasing drought stress over two weeks by sealing the container and allowing transpiration only via the leaves. Water contents were mapped by relaxometric imaging using a multi-slice- multi-echo method (MSME). Additionally, the root system architecture was imaged by a 3D fast spin echo method (RARE) with high resolution. 


\section{MATERIAL AND METHODS}

\section{Plant Setup}

$10 \mathrm{~cm}$ high cylindrical Perspex containers with $8 \mathrm{~cm}$ internal diameter were used for all experiments. Each was equipped with two $0.8 \times 15 \mathrm{~cm}$ cylindrical marker tubes filled with glass beads and saturated with $5 \mathrm{mM} \mathrm{NiCl}_{2}$ solution as internal water content standard $(\theta=0.39)$ and for coregistration of the MRI images recorded at different dates. The containers were filled about $10 \mathrm{~cm}$ high with medium sand $(0.2-0.7 \mathrm{~mm})$ and a $2 \mathrm{~cm}$ top layer of peat. Ricinus seeds have been planted in the peat layer, see Fig. (1). After germination they grew for three weeks. Then the container was saturated slowly from bottom and sealed on both sides so that transpiration proceeded only via the leaves. Three individual plants have been grown, of which one is chosen here for further evaluation of the data.

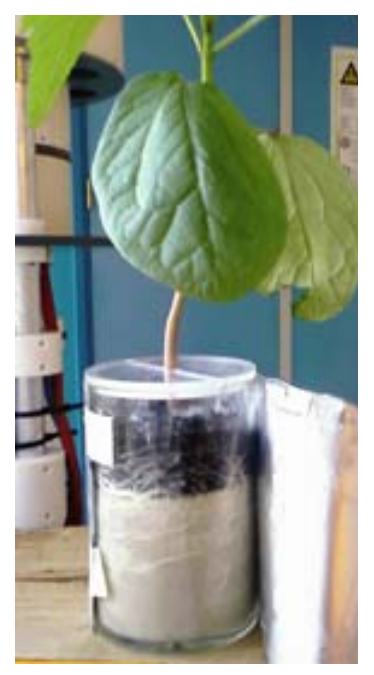

Fig. (1). Ricinus plant in the container immediately before measuring.

Fig. (2) shows the gravimetrically determined water content changes with time after saturation and sealing on day- 0 . The small vertical arrows indicate the dates of the MRI experiments.

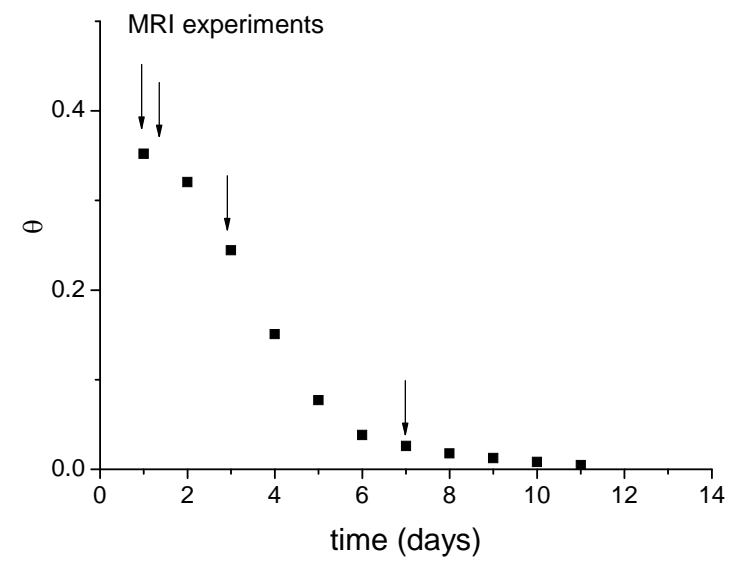

Fig. (2). Gravimetric total water content in the container after saturation and sealing. The arrows indicate the dates of the MRI experiments.

\section{MRI Hardware \& Protocols}

All MRI experiments have been performed at the Wageningen 3T scanner (127.9 MHz, $\left.\mathrm{G}_{\max }=0.27 \mathrm{~T} / \mathrm{m}\right)$, operated by a Bruker console. The root architecture was determined with high spatial resolution using a 3D-RARE pulse sequence with a turbo factor of $16 . T_{\mathrm{E}}$ was $6.7 \mathrm{~ms}$ for the first echo and $4.3 \mathrm{~ms}$ for the subsequent 15 echos, $T_{\mathrm{R}}=$ $1.5 \mathrm{~s}$. The Voxel size was isotropic $0.78 \mathrm{~mm}$. Quantitative water content mapping was performed by a multislicemultiecho sequence (MSME)[13]. The simplified timing diagram is displayed in Fig. (3). In contrast to conventional single-echo pulse sequences it monitors a sequence of many echoes $n_{\mathrm{E}}$ for each voxel, addressed by the slice selective initial $90^{\circ}$ pulse, the phase encoding ( $p e$ ) and the read-out (ro) gradients. Before further analysis the data, which have been recorded in the complex space, were phase corrected so that the information was only in the real part, and the imaginary part was ignored. This is especially important for biexponential fitting [16]. The signal intensity for biexponential decay due to transversal relaxation under the condition for sufficiently long repetition time is given by Eq. 1 .

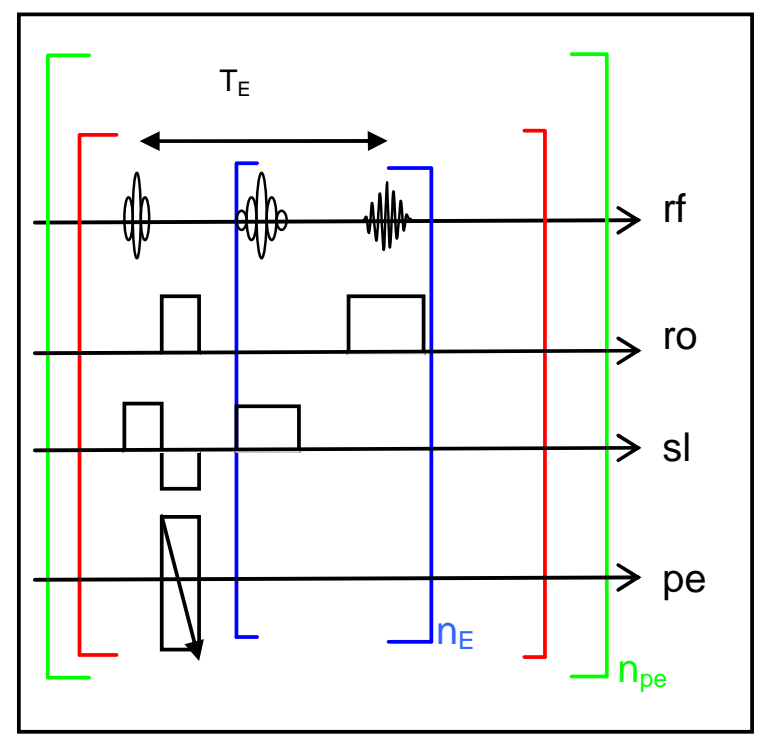

Fig. (3). simplified MSME imaging timing diagram. $T_{E}$ is echo time, $n_{E}$ the number of echoes, $r f, r o, s l$, and pe refer to rf-channel, read-out, phase-encoding, and slice selection gradients, respectively. Further details see text.

$S\left(n_{E} T_{E}\right) \propto S_{0 f} \exp \left(\frac{-n_{E} T_{E}}{T_{2, f}}\right)+S_{0 s} \exp \left(\frac{-n_{E} T_{E}}{T_{2, s}}\right)$

$S_{0}=S_{0, \mathrm{f}}+S_{0, \mathrm{~s}}$

$n_{\mathrm{E}}$ and $T_{\mathrm{E}}$ are the number of echoes and echo time, respectively. $S_{0, \mathrm{f}}, S_{0, \mathrm{~s}}, T_{2, \mathrm{f}}$, and $T_{2, \mathrm{~s}}$ are the amplitudes and transversal relaxation times of the fast and slow processes, respectively. So, by extrapolation the quantity $S_{0}=S_{0, \mathrm{f}}+S_{0, \mathrm{~s}}$ is obtained, which is proportional to the spin density and the water content in individual voxels. We used the following imaging parameters: matrix size $32 \times 32$ and $3 \mathrm{~mm}$ slice thickness $(10 \times 10 \times 9.6 \mathrm{~cm})$. Slices were addressed in interleaved 
mode. $T_{\mathrm{E}}=6.76 \mathrm{~ms}, n_{\mathrm{E}}=128, T_{\mathrm{R}}=30 \mathrm{~s}$. The water content maps are obtained by calibration of the $3 \mathrm{D}$ amplitude maps on $S_{0}$ of the voxels of the calibration tubes. All data evaluation steps were performed by the IDL programming system.

\section{RESULTS AND DISCUSSION}

Fig. (4) shows some exemplary horizontal and vertical slices through the ricinus container. Plotted are the intensity of the first echo and relaxation curves of selected voxels indicated by the yellow arrows. Although the resolution is limited to $3 \mathrm{~mm}$, we observe structural details in the container. In Fig. (4a) the yellow arrow points to the calibration tube. In Figs. (4b) to (4e) roots are clearly resolved, as well as the peat layer in the upper part of the container, which is much more heterogeneous than the sand packing below. Also the signal intensity is much higher. In Fig. (4d) a root is indicated and in Fig. (4e) the arrow points to the onset of the shoot inside the peat layer. In the lower parts of the subfigures relaxation curves in selected voxels are shown for a) in the marker, b) and c) in bulk soil on day-1 and day- 6 after saturation, d) is from a root voxel and e) from a shoot voxel.
One should take into account that the voxel size is greater than the dimension of the roots, so that the intensity is composed of both signals from root and the immediately surrounding soil. This partial volume effect is responsible for the lower signal intensity in the root-voxel compared to the shoot voxel, since the shoot is much thicker than the roots.

In most voxels bimodal transverse relaxation was observed, so Eq. 1 was fitted to all voxels. From that we obtained amplitude, $\mathrm{T}_{2, \mathrm{f}}$ and $\mathrm{T}_{2, \mathrm{~s}}$ maps. This is exemplarily shown for 30 vertical slices in Fig. (5) for the plant on day-1. The amplitudes are quite uniform over the sand packing, with exception of the slices 2 and 3 from bottom. Clearly distinguishable is the peat layer in the upper $2 \mathrm{~cm}$. It is characterized by high amplitudes reflecting high water contents and by short fast transverse relaxation times $T_{2, \mathrm{f}}$, which are in the range between 3 and $8 \mathrm{~ms}$, whereas the slow relaxation time $T_{2, \mathrm{~s}}$ is about $80 \mathrm{~ms}$ and comparable to the range of the sand packing. The second feature is the inner and outer marker tubes in images 16 to 18 and 26 to 30 , respectively, characterized by moderate amplitudes and long relaxation times $\left(T_{2, \mathrm{f}} \cong 20 \mathrm{~ms}, T_{2, \mathrm{f}} \cong 200 \mathrm{~ms}\right)$. Also voxels including roots a)
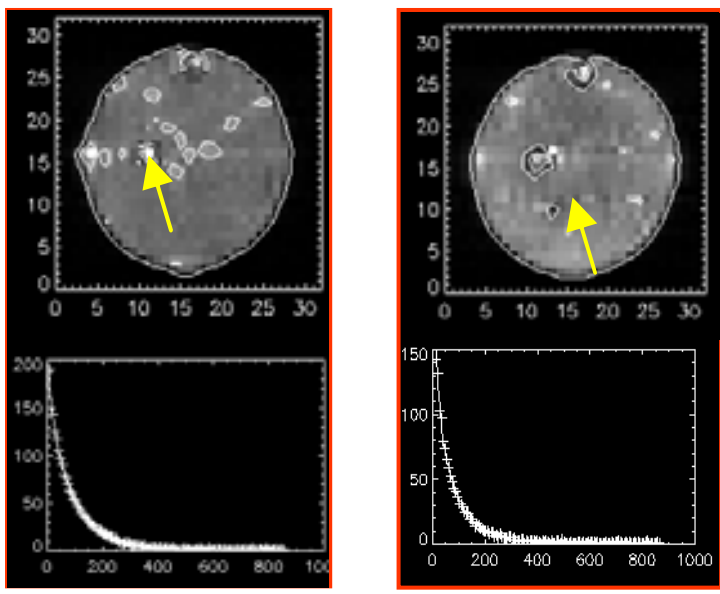

d)

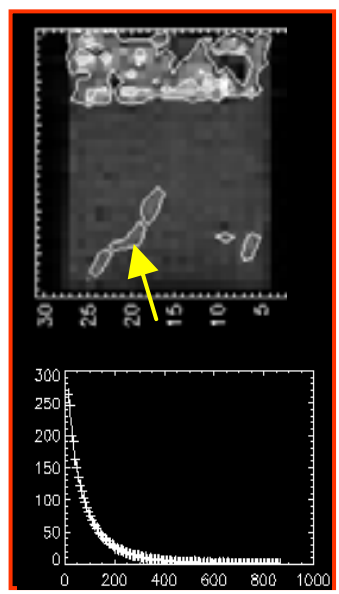

c)

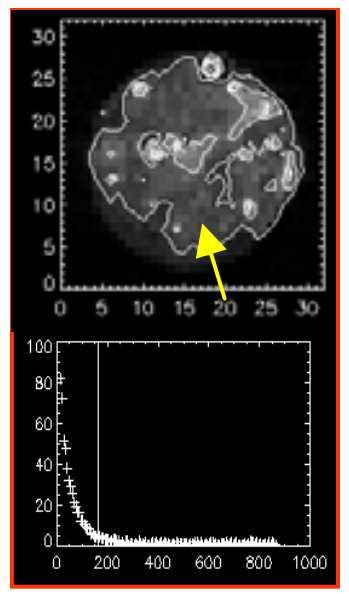

e)

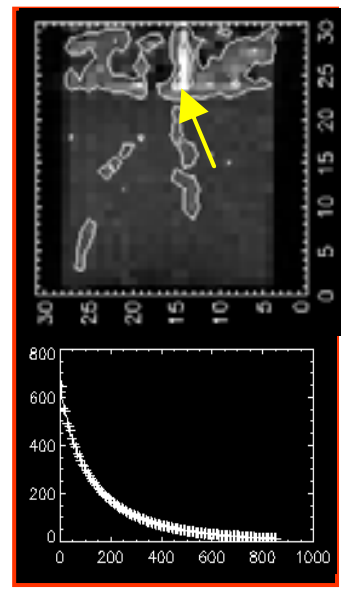

Fig. (4). Exemplary MSME-MRI images of selected slices of the ricinus container. a) axial slice near bottom at day-1, b) and c) axial slices near top on day-1 and day-6, resp. d) and e) vertical slices showing roots and shoots. The arrows indicate individual voxels for which the $T_{2}$ decay curves are shown in the lower parts of the subfigures. 

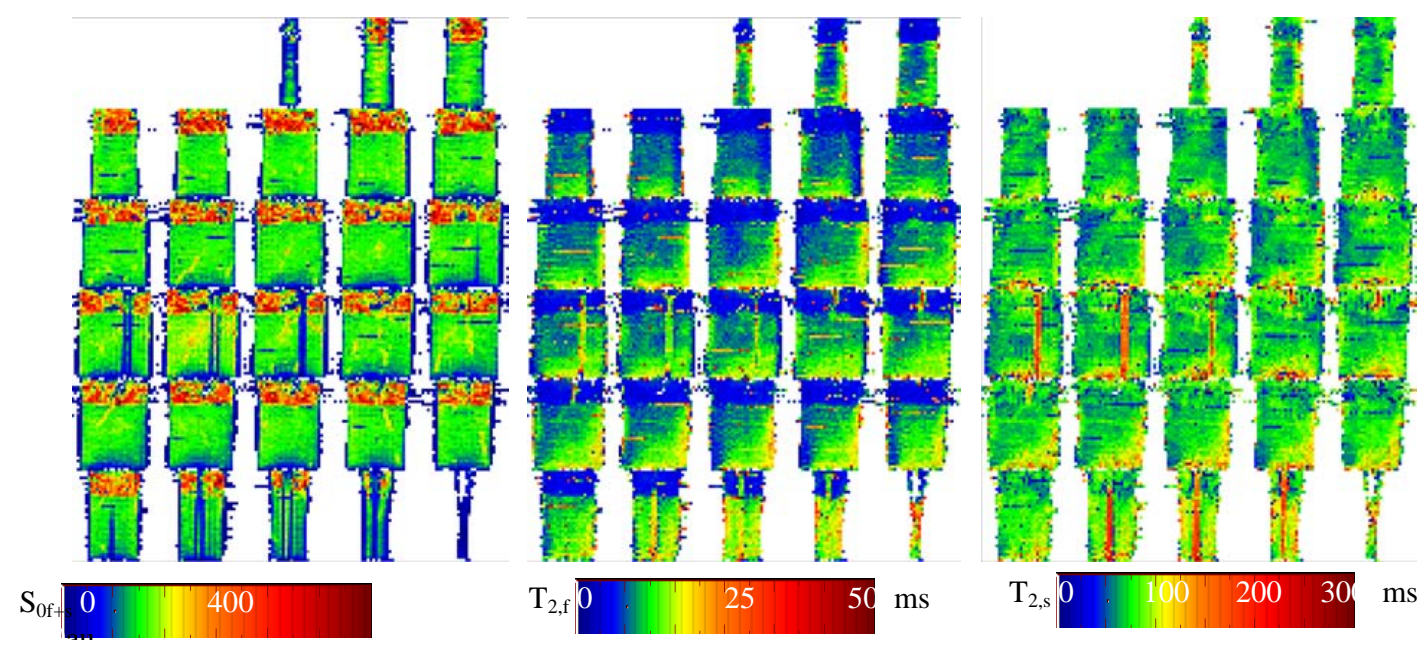

Fig. (5). Total amplitude $S_{0}, T_{2, \mathrm{f}}$ and $T_{2, \mathrm{~s}}$ maps obtained for the ricinus container on day-1 by fitting of Eq. 1 to the relaxation curve in all voxels. Shown are 30 from 32 vertical slices, FOV is $10 x 10 \mathrm{~cm}$, matrix size $32 \times 32$. The slices are enumerated 1 to 30 from top-left to bottomright.

are visible in the amplitude maps of the central vertical slices, whereas they are not clearly identifiable in the relaxation time maps. However, it should be noted that in the areas with highest root densities, e.g. slices 17 and 18, both fast and slow relaxation times are faster $\left(T_{2, \mathrm{f}} \cong 8 \mathrm{~ms}, T_{2, \mathrm{f}} \cong 50 \mathrm{~ms}\right)$, than in the outer regions of the container $\left(T_{2, \mathrm{f}} \cong 14 \mathrm{~ms}, T_{2, \mathrm{f}} \cong\right.$ $80 \mathrm{~ms})$. The nature of the fast and slow relaxation time is not yet clear. Assuming that the Brownstein-Tarr model is valid, transverse relaxation is accelerated in small pores due to frequent wall contacts of diffusing water molecules in the neighbourhood of paramagnetic centres. Second, transverse relaxation is accelerated by diffusion in internal gradients [14]. This might explain the frequently observed bimodal relaxation in natural porous media, but this requires more detailed investigations, which exceed the frame of this work.

The central aim of this work is the determination of water content changes due to root system activity. As the next step we have calculated the water content $\theta(\mathrm{x}, \mathrm{y}, \mathrm{z})$ by calibration of the total amplitude maps on the marker tubes with $\theta=$ 0.39 . This evaluation procedure is validated by comparing the average gravimetric water contents of the plant container with the MRI determined water content.

Fig. (6) shows that MRI-water content profiles, which have been obtained by integration over horizontal agree well with the average gravimetric water contents. This proves the validity of the entire measurement and evaluation procedure. Examples of the water content map for slice 19 on day- 1 and day-6 are shown in Fig. (7). One can clearly recognize the roots and the peat layer on the top. The soil appears quite homogeneous with some enrichment of water near the bottom. On day- 6 the whole system is much drier, the average water content in the sand is about $\theta=0.05$. The top peat layer is also much drier, so that the onset of the shoot becomes visible. A striking point is that the voxels containing roots also appear much drier (blue colour $v s$. red colour on day-1), although one might expect that the roots do not loose water in significant amounts unless the plant is wilted. So they should appear white just like the shoot. The reason for this is that the voxel size is with $3 \mathrm{~mm}$ significantly larger than the root diameter, so the lower intensity is due to a partial volume effect; one observes average water content composed of root and surrounding soil.

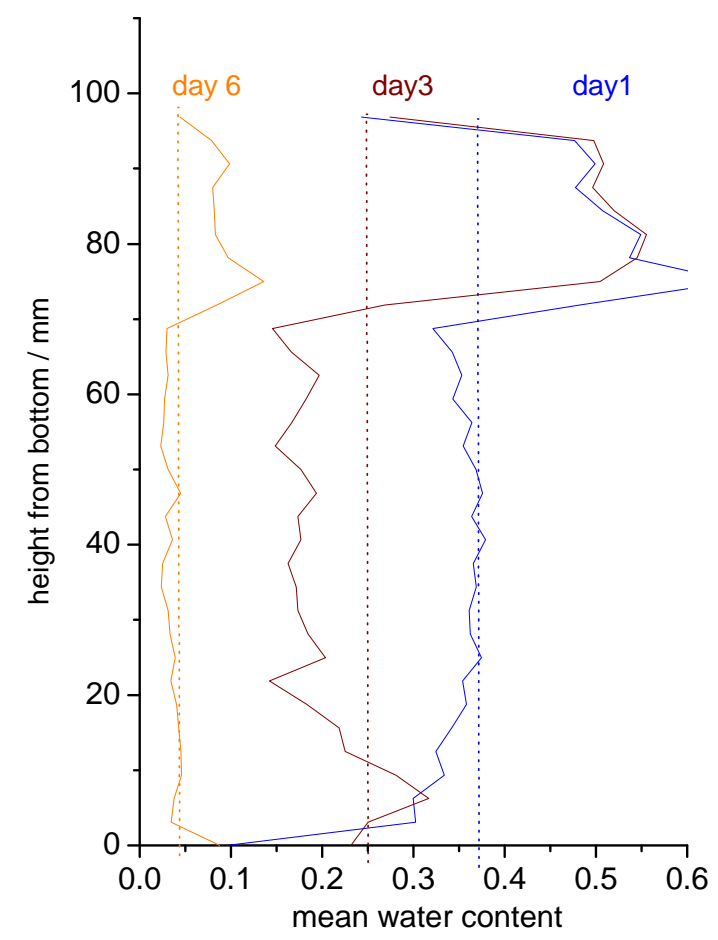

Fig. (6). Vertical water content profiles of the ricinus-root soil system, calculated by integration over horizontal slices from the water content maps.

For a comparison of the root system with the water content maps we have also recorded 3D images of only the roots by MRI with a 3D RARE sequence. This is presented in Fig. (8), where voxels with intensities above a threshold of $25 \%$ of the maximum are enclosed by an isosurface. The peat layer is cut off. 


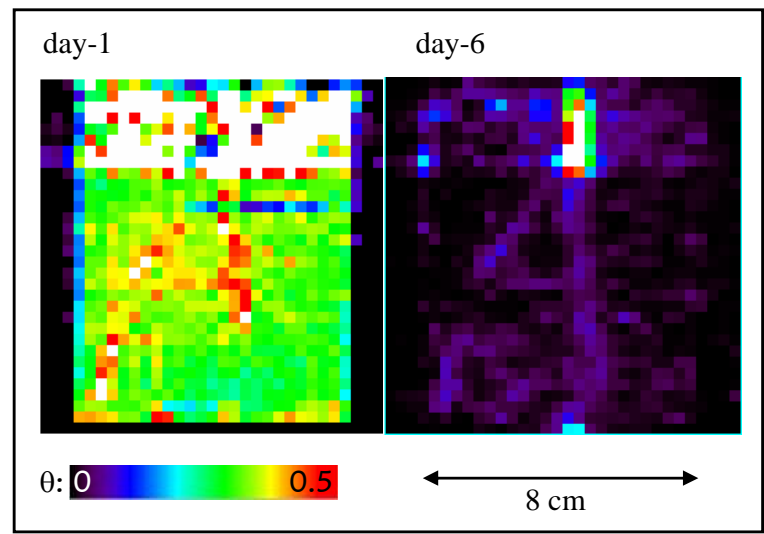

Fig. (7). Selected water content maps of the ricinus-root soil system. Shown is a central vertical slice on day 1 and day 6 after saturation and sealing.

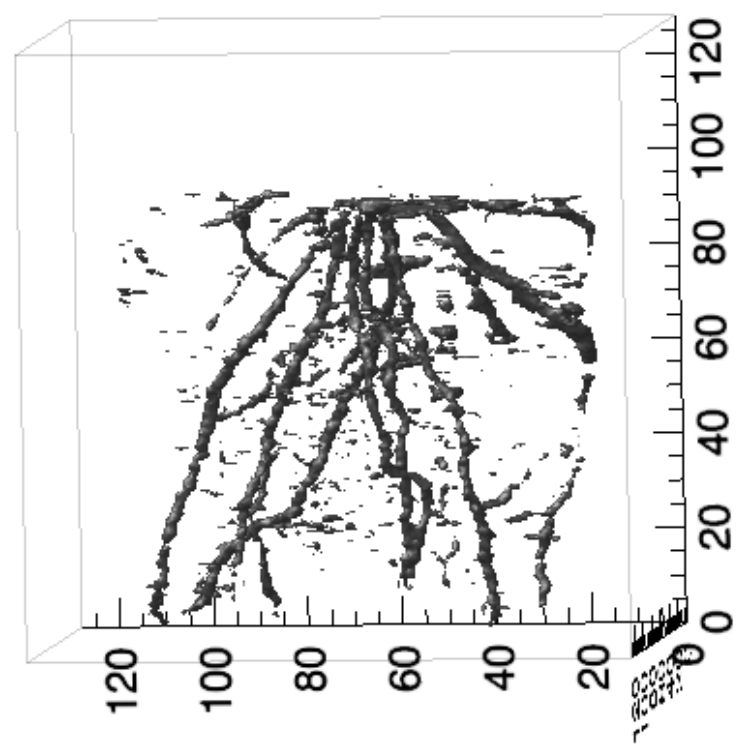

Fig. (8). 3D MRI-RARE image of the ricinus root system on day-1. The scales are voxels, voxel size is $0.078 \mathrm{~mm}$, matrix size $128 \times 128 \times 128$, FOV 10x10x9.6 cm.

Beneath the roots spots are also visible, which do not belong to roots but are sites with locally high water content. For an easier comparison to the water content maps Fig. (9) presents an excerpt from the 3D image in Fig. (8), which corresponds to the vertical slice shown in Fig. (7).

The maximum width of the main roots is 3 voxels, i.e. $2.3 \mathrm{~mm}$ which is much smaller than the voxel size for the water content maps. Going back to Fig. (6) one can see that for example the central root appears about 3 voxels wide, i.e. about $9 \mathrm{~mm}$. Since this is much larger than the root thickness itself this strongly indicates wetter zones around the roots. Such behaviour has also been observed by others $[17,18]$.

The final step of the evaluation is the calculation of water content difference maps in order to see where the root system has taken up water preferentially. In Fig. (10) the root

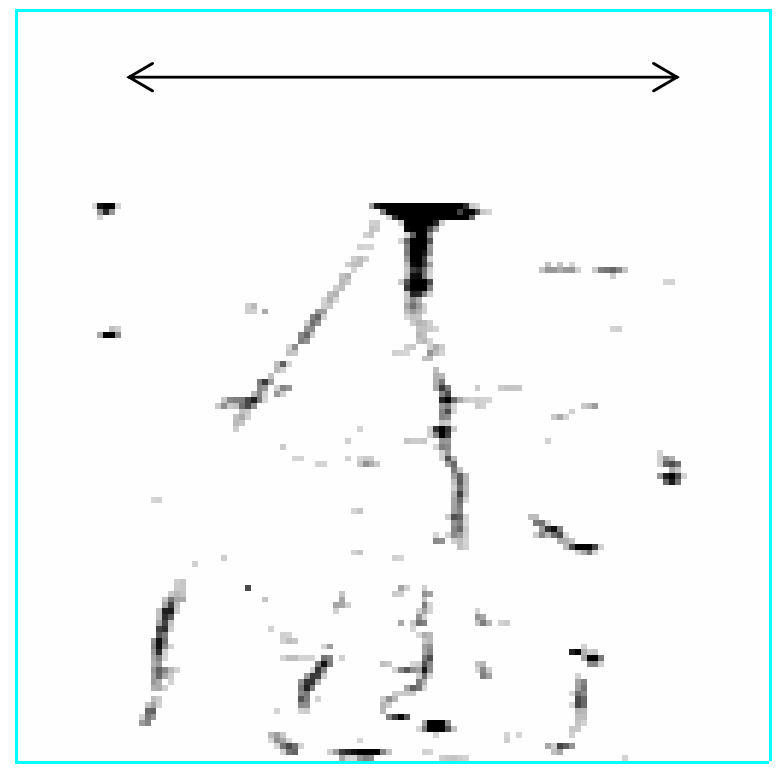

Fig. (9). Central vertical slice of the MRI-RARE image of the ricinus root system.

system architecture is overlaid with four axial slices of the water content differences between day-1 and day-6. The water content change was greatest in the upper central part of the soil core, whereas near bottom if remained wetter. Also in the neighbourhood of the roots greater changes of the water content is observable. This indicates that the hydraulic conductivity is decreased so far that the water uptake by the roots is not yet compensated instantaneously by flux from more distant regions.

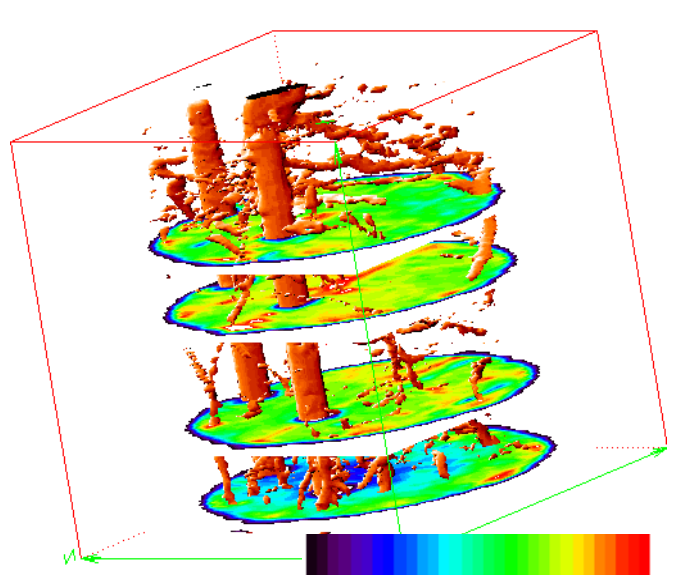

Fig. (10). ricinus-root soil system. Difference of water content $\Delta \theta$ between day- 6 and day-1, overlayed by the root system, obtained from the RARE MRI measurement. (The long cylinders in the left half are the marker tubes).

\section{CONCLUSIONS}

MRI was used for both imaging of the root system architecture and the water contents in a three week old ricinus 
grown in natural sand. For the determination of the water content it is advantageous to use a relaxometric multi-echo method since this yields relaxation time maps as well as amplitude maps, from which water contents can be obtained by calibration on internal reference samples. The results of these investigations agree with those we have obtained in a previous work by the application of a single point imaging method (SPRITE) [10]. But in contrast to that MSME is faster, since the total measurement time single point imaging methods scales with the cube of the resolution, echo methods only with the square. The second advantage of MSME is the determination of $\mathrm{T}_{2}$ maps which reflect local soil properties, whereas SPI determines $\mathrm{T}_{2}{ }^{*}$, which is mainly controlled by magnetic field inhomogeneities. On the other hand multiecho imaging requires $T_{2}$ relaxation times greater than some milliseconds, since the minimum $\mathrm{T}_{\mathrm{E}}$ is about $1.5 \mathrm{~ms}$. Since in many natural soils $\mathrm{T}_{2}$ can be faster, multi-echo imaging would show no signal, so that single point imaging must be applied.

By the analysis of the water content maps we found that in the neighbourhood of the roots zones with increased water content exist also when the total water content decreased to $\theta$ $<0.05$. Anyway, the water content change in some these areas was greater than in the bulk soil, which can be explained by initially increased water content. The next step of the analysis will be soil physical modelling of root water uptake processes, which requires a continuous representation of the root system (skeletisation). For this purpose the quality of the root system imaging should be improved in terms of a better discrimination between soil and roots and the closing of apparent gaps along the roots strands. This could by performed by either other imaging methods or image processing techniques.

\section{ACKNOWLEDGEMENTS}

The authors are grateful to EU for funding the NMR experiments at the Wageningen NMR Centre (WNMRC07001), to the DFG (SFB TR-32, PO-746-2/1) for financial support, and Beate Uhlig, ICG-3, Research Centre Jülich for growing the plants.

\section{REFERENCES}

[1] Waisel Y, Eshel A, Kafkafi U. Plant roots: the hidden half. Marcel Dekker: New York 1991

[2] Javaux M, Schröder T, Vanderborght J, Vereecken H. Use of a three-dimensional detailed modeling approach for predicting root water uptake. Vadose Zone J 2008; 7: 1079-88.
[3] Doussan C, Pierret A, Garrigues E, Pagès L. Water uptake by plant roots: II - Modelling of water transfer in the soil root-system with explicit account of flow within the root system: comparison with experiments. Plant Soil 2006; 283: 99-117.

[4] Garrigues E, Doussan C, Pierret A. Water uptake by plant roots: I Formation and propagation of a water extraction front in mature root systems as evidenced by $2 \mathrm{D}$ light transmission imaging. Plant Soil 2006; 283: 83-99.

[5] Perret J, Al-Belushi ME, Deadman M. Non-destructive visualization and quantification of roots using computed tomography. Soil Biol Biochem 2007; 39: 391-9.

[6] Tumlinson LG, Liu HY, Silk WK, Hopmans JW. Thermal neutron computed tomography of soil water and plant roots. Soil Sci Soc Am J 2008; 72: 1234-42.

[7] Moradi AB, Oswald S, Massner JA, Pruessmann KP, Robinson $\mathrm{BH}$, Schulin R. Dynamic of nickel in the rhizosphere of Berkheya coddii using magnetic resonance imaging. Geophys Res Abstr 2008a; 10: EGU2008-A-08398.

[8] Nestle N, Baumann T, Niessner R. Magnetic resonance imaging in environmental science. Environ Sci Technol 2002; 36: 154A-60A.

[9] MacFall JS, Van As H. Magnetic Resonance Imaging of Plants. In: Shachar-Hill Y, Pfeffer PE, Eds. Nuclear magneit resonance in Plant Biology. Rockville, MD: American Society of PlantPhysiologists 1996.

[10] Pohlmeier A, Oros-Peusquens AM, Javaux M, et al. Changes in soil water content resulting from ricinus root uptake monitored by magnetic resonance imaging. Vadose Zone J 2008; 7: 1010-7.

[11] Pohlmeier A, Oros-Peusquens AM, Javaux M, Menzel MI, Vereecken H, Shah NJ. Investigation of water content and dynamics of a Ricinus root system in unsaturated sand by means of SPRITE and CISS: correlation of root architecture and water content change. Magn Reson Imaging 2007; 25: 579-80.

[12] Stingaciu LR, Pohlmeier A, Blümler P, et al. Characterization of unsaturated porous media by high-field and low-field NMR relaxometry. Water Resour Res 2009; 45: W08412.

[13] Edzes HT, van Dusschoten D, Van As H. Quantitative T-2 imaging of plant tissues by means of multi-echo MRI microscopy. Magn Reson Imaging 1998; 16: 185-96.

[14] Barrie PJ. Characterization of porous media using NMR methods. Ann Rep NMR Spectrosc 2000; 41: 265-316.

[15] Stingaciu LR, Pohlmeier A, Blümler P, Weihermüller L, Stapf S, Vereecken $\mathrm{H}$. Characterization of unsaturated porous media by high-field and low-field NMR relaxometry. Water Resour Res 2009; 45: W08412.

[16] Van der Weerd L, Vergeldt F, de Jager PA, Van As H. Evaluation of algorithms for analysis of NMR relaxation decay curves. Magn Reson Imaging 2000; 18: 1151-8.

[17] Carminati A, Moradi A, Vetterlein D, et al. Dynamics of soil water content next roots: the role of the rhizosphere. Plant Soil 2009: (in print).

[18] Haber-Pohlmeier S, Van Dusschoten D, Stapf S. Waterflow visualized by tracer transport in root-soil-systems using MRI. Geophys Res Abstr 2009; 11: EGU2009-8096.

(C) Pohlmeier et al.; Licensee Bentham Open.

This is an open access article licensed under the terms of the Creative Commons Attribution Non-Commercial License (http://creativecommons.org/licenses/by-nc/3.0/) which permits unrestricted, non-commercial use, distribution and reproduction in any medium, provided the work is properly cited. 